\title{
Thermal expansion and Grüneisen parameters of amorphous silicon: A realistic model calculation
}

\author{
Jaroslav Fabian and Philip B. Allen \\ Department of Physics, State University of New York at Stony Brook, Stony Brook, New York \\ $11794-3800$
}

\begin{abstract}
Using a realistic model, the mode Grüneisen parameters $\gamma$ and the temperature dependent coefficient of linear thermal expansion $\alpha(T)$, are calculated for amorphous silicon. The resulting $\gamma$ values differ from the crystalline case in having all diversity suppressed, except for a minority of high-frequency localized and low-frequency resonant modes. The latter have very large, mostly negative $\gamma$ (up to -31 ), caused by volume driven internal strain. As a result, the values for $\alpha(T)$ are lower than those of crystalline silicon and are sample dependent.
\end{abstract}

$65.70 .+\mathrm{y}, 63.50 .+\mathrm{x}$

Typeset using REVTEX 
Unlike thermal conductivity and specific heat, which have "universal" features, thermal expansion is a special property of each glass. The coefficient of linear expansion $\alpha$ can be either positive or negative, with magnitude sensitive to sample preparation methods [1]. This is true even at very low temperatures $(T \lesssim 1 \mathrm{~K}$ ), where $\alpha$ is believed to be associated with tunneling modes [2] and large dispersion of $\gamma$ values is found [3].

Here we present an analysis of $\alpha(T)$ for an atomistic model of amorphous silicon, an important electronic material. The value of $\alpha(T)$ has been measured only at $\mathrm{T}=383 \mathrm{~K}$ [4], making theoretical modelling particularly useful. Our calculation shows that the value of $\alpha(T)$ is lower than that of crystalline silicon. Deviations from the crystalline values are more dramatic at lower temperatures, and are caused by volume driven internal strain, which makes the thermal expansion sample dependent. The predicted Grüneisen parameters display a surprising simplicity, which we interpret as evidence for special, possibly generic, properties of vibrations in glasses. Specifically, we find that the majority of modes which are neither localized nor ballistically propagating (we call them "diffusons"), have a property of global indistinguishability, whereas high-frequency localized and low-frequency resonant modes are distinguished by the special structural imperfections at which they have largest amplitudes. In particular, the resonant modes have a considerable dispersion of $\gamma$ values (from -31 to 4), consistent with our finding that internal strain is largest at the centers of these modes. Below $1 \mathrm{~K}$, very large average magnitudes of $\gamma(|\gamma| \gtrsim 10)$ were measured in many glasses [3] ("normal" values are about one). Although the size of our model does not allow us to go as low as $1 \mathrm{~K}$, we show that the resonant modes provide a likely origin of this anomaly. Finally, we establish a close link between the mode Grüneisen parameters and the mode bond-stretching character.

Insofar as a complete set of normal modes can be defined (i.e., the modes decay on timescales much larger than their periods), one expects vibrational entropy $S$ to be well approximated by $S=k_{B} \sum_{a}\left[\left(n_{a}+1\right) \log \left(n_{a}+1\right)-n_{a} \log n_{a}\right]$, where $n_{a}=\left(\exp \left(\hbar \omega_{a} / k_{B} T\right)-1\right)^{-1}$ is the average equilibrium occupation number of the mode of frequency $\omega_{a}, a$ being a counting label going from 1 to $3 N . N$ is the number of atoms and $k_{B}$ is the Boltzmann constant. The 
frequency $\omega_{a}(V, T)$ may depend on both volume $V$ and temperature. Using the standard thermodynamic relation $3 \alpha=\kappa_{\mathrm{T}}(\partial S / \partial V)_{T}$, with isothermal compressibility $\kappa_{\mathrm{T}}$, we get (see, e.g., Ref. [5])

$$
\alpha(T)=\frac{\kappa_{\mathrm{T}}}{3 V} \sum_{a} c_{a} \gamma_{a} .
$$

Here $c_{a}=k_{B}\left(\hbar \omega_{a} / k_{B} T\right)^{2} n_{a}\left(n_{a}+1\right)$ is the specific heat of a harmonic oscillator, and $\gamma_{a}$, given by $\gamma_{a}=-\partial \log \omega_{a} / \partial \log V$, are known as the "mode Grüneisen parameters"; they measure how sensitive the vibrational eigenfrequencies are to the change of volume. Their knowledge is essential not only for $\alpha(T)$, but also for the interpretation of the internal friction and sound attenuation experiments [6.7].

Perturbation theory (see, e.g., Ref. [8]) gives the following formula for $\gamma_{a}$,

$$
\begin{aligned}
\gamma_{a} & =-\frac{1}{6 \omega_{a}^{2}} \sum_{i j k} \sum_{\alpha \beta \gamma} \Phi_{\alpha \beta \gamma}^{i j k} \frac{e_{i \alpha}^{a *} e_{j \beta}^{a}}{\sqrt{M_{i} M_{j}}}\left(r_{k \gamma}+\bar{r}_{k \gamma}\right), \\
\bar{r}_{i \alpha} & =-\sum_{a} \frac{1}{\omega_{a}^{2}} \sum_{j k} \sum_{\beta \gamma} \Phi_{\beta \gamma}^{j k} \frac{e_{i \alpha}^{a *} e_{j \beta}^{a}}{\sqrt{M_{i} M_{j}}} r_{k \gamma} .
\end{aligned}
$$

Here $\Phi_{\alpha \beta}^{i j}\left(\Phi_{\alpha \beta \gamma}^{i j k}\right)$ are the coefficients of the quadratic (cubic) terms of the Taylor expansion of potential energy in terms of the displacements $u_{i \alpha}$ of atoms $i$ from equilibrium in the direction $\alpha=(x, y, z)$, and $r_{i \alpha}$ is the position vector of the $i$ th atom. The vibrational eigenvectors $e_{i \alpha}^{a}$ are normalized $\left(\sum_{i \alpha}\left|e_{i \alpha}^{a}\right|^{2}=1\right)$, and $M_{i}$ is the mass of atom $i$. The sums in Eq. 2 and are over all atoms. Because of periodicity, in a crystal the label $a$ can be written as $(\mathbf{Q}, \lambda)$, denoting wave vector and polarization. The eigenvectors $e_{i \alpha}(\mathbf{Q}, \lambda)$ are proportional to $e^{i \mathbf{Q} \cdot \mathbf{r}_{i}} / \sqrt{N}$, and the resulting crystalline phase coherence allows a simplification of the sum in Eq. 2 to the neighborhood of a single small unit cell. By contrast, normal modes in a glass have no a priori quantum numbers, and quantitative insight is best achieved by numerical diagonalization for finite models.

If a solid is subject to an infinitesimal homogeneous isotropic strain $\epsilon$, its atomic coordinates change to $r_{i \alpha}^{\prime}=r_{i \alpha}(1+\epsilon)$, and volume to $V^{\prime}=V(1+3 \epsilon)$. Unless atoms in the solid are fixed by symmetry, $r_{i \alpha}^{\prime}$ are not the equilibrium coordinates of the solid with volume $V^{\prime}$. 
This happens in glasses. After the strain is applied, the atoms relax to new equilibrium positions $r_{i \alpha}^{\prime}+\epsilon \bar{r}_{i \alpha}$. This is the origin of the internal strain (Eq. 3) occuring during the thermal expansion of glasses (and crystals with atoms not in centers of symmetry [1]). In silicon crystal $\bar{r}_{i \alpha} \equiv 0$.

The interactions between silicon atoms are represented by the Stillinger-Weber (SW) potential [9], which performs well when applied to elastic and vibrational properties of different silicon phases [10 12]. For the atomic coordinates of amorphous silicon we take the model introduced and described in detail in Ref. [11. The algorithm by Wooten, Winer, and Weaire (WWW) 13 creates a random network structure of silicon atoms, which are further relaxed to a local minimum of the SW potential. Here we use 1000 atoms arranged in a cube of side $27.543 \AA$, with periodic boundary conditions. The energy/atom of this model is $-4.102 \mathrm{eV}$, which is $\approx 5 \%$ higher than the energy/atom of crystalline silicon with $\mathrm{SW}$ potential. Also, the model agrees very well with the neutron structure factor $S(Q)$ measured by Kugler et al. [14].

To calculate $\alpha(T)$ we need the values for $\kappa_{\mathrm{T}}$. The corresponding perturbation formulas for $\kappa_{\mathrm{T}}$, which take into account internal strain, can be found in Ref. [15]. We obtain the value $1.13 \times 10^{-12} \mathrm{~cm}^{2} / \mathrm{dyn}$ for the present model of amorphous silicon. For crystalline silicon, the SW potential gives the value of $0.986 \times 10^{-12}$ [16], agreeing well with the experimental value of $1.02 \times 10^{-12}[16$. Also, for silicon crystal, we must sum over the $\mathbf{Q}$ points, which we do using the tetrahedron method with 1772 tetrahedra in the irreducible wedge of the Brillouin zone. The results are shown in Fig. 1, together with the measured data.

Compared with experiment [17], $\alpha(T)$ of silicon crystal is reproduced very well at $T \gtrsim 200$ K. At lower $T$ the SW potential does not reproduce the observed negative values of $\alpha$. Negative $\alpha$ has been succesfully explained [18] by negative values of $\gamma$ for the low energy transverse acoustic (TA) branch. As shown in Fig. (2(A), our $\gamma$ values are too weakly negative in this regime. In Fig. 1 it is predicted that $\alpha(T)$ for amorphous silicon is somewhat lower than $\alpha(T)$ for the crystal. For comparison, Fig. 1 also shows $\alpha(T)$ without considering internal strain $\left(\bar{r}_{i \alpha}\right.$ in Eq. 2 is set to zero), and the calculation based on a 216 atom model 
of amorphous silicon. Internal strain reduces the values of $\alpha$ by almost $30 \%$ at high $T$. At lower $T$ the values become negative. The model dependence is also clear. The 216 atom model is based on the same WWW algorithm and SW potentials, but is more topologically constrained (e.g., no four-fold rings are allowed), and has lower energy/atom (by $\approx 0.5 \%$ ) and higher density (by $\approx 3 \%$ ) than the 1000 atom model. Its $\alpha(T)$ is higher and closer to the crystalline case and the measured value. This is not surprising. Measurements on silica [1.19 showed that annealing history (or density) markedly changed its thermal expansion at all temperatures. For example, pure $\mathrm{SiO}_{2}$ aged at $1400^{\circ} \mathrm{C}$ and quenched, has $\alpha(T)$ at high $T$ lower by up to $50 \%$ than that slowly aged at $1000^{\circ} \mathrm{C}$. Our calculation predicts that future experiments should see similar behavior in amorphous silicon as well.

To understand the behavior of $\alpha(T)$ one has to look at the frequency dependence of Grüneisen parameters. The values of $\gamma$ for silicon crystal, for more than 1000 randomly chosen $\mathbf{Q}$ points from the irreducible wedge of the Brillouin zone, are in Fig. 2(A). Since there is a degenerate surface in $\mathbf{Q}$ space for a given $\omega, \gamma(\mathbf{Q}, \lambda)$ has a distribution of values at each $\omega$. These values are further split according to the branch $\lambda$ of corresponding phonons. Particularly striking polarization effects appear at low $\omega$, where TA phonons form a distinct broad band of $\gamma_{a}$.

It is instructive to compare Fig. 2(A) with Fig. 2(B), where we plot the mode bondstretching parameter $S_{a}$,

$$
S_{a}=\left[\frac{\sum_{<i, j>}\left|\left(\mathbf{u}_{i}^{a}-\mathbf{u}_{j}^{a}\right) \cdot \mathbf{n}_{i j}\right|^{2}}{\sum_{<i, j>}\left|\mathbf{u}_{i}^{a}-\mathbf{u}_{j}^{a}\right|^{2}}\right]^{\frac{1}{2}},
$$

introduced in Ref. [20] (we modified slightly the original formula which did not yield the same $S_{a}$ for different but degenerate $a$ ). The summation is over all atom pairs $\langle i, j\rangle$, where $j$ is within $2.8 \AA$ (the distance where the first nearest neighbor peak in the pair correlation function of amorphous silicon ends [14]) from $i$, and $\mathbf{n}_{i j}$ is the unit vector in the direction of the bond $\langle i, j\rangle$. When $S_{a}$ is close to one, mode $a$ is mostly bond-stretching, while values closer to zero indicate bond-bending modes. The similarity between the two figures is striking. Except at very low $\omega$, one is tempted to write " $\gamma_{a} \approx$ const $\times S_{a}$." The 
reason for such close relation is that anharmonicity, reflected in $\gamma_{a}$, is much greater in the bond-stretching part of the interatomic interaction than in the bond-bending part.

We now discuss the behavior of $\gamma_{a}$ in amorphous silicon, whose vibrational states were discussed earlier [11, 12]. At low frequencies $(\lesssim 15 \mathrm{meV})$ the modes are propagons, acousticphonon-like vibrations propagating ballistically for distances greater than their wavelength. Some of the propagons are resonantly trapped at certain places in the sample, with reduced amplitude elsewhere (but not exponential decay as in a localized state); but at high $\omega$ there is no continuum of extended states to decay into, and vibrational amplitudes decay exponentially with distance. Properties of these resonant modes were studied in different glassy systems [21], and for our model will be reported elsewhere [22]. Modes between 15 $\mathrm{meV}$ and $71 \mathrm{meV}$ are diffusons, extended states carrying energy diffusively. Since they form the majority of the spectrum, diffusons dominate the thermal properties of amorphous silicon at temperatures from several kelvins up to the melting point. Above $\omega_{c} \approx 71 \mathrm{meV}$, the mobility edge, the modes are locons, localized states.

The calculated $\gamma_{a}$ are shown in Fig. 3(A). Three regions can be clearly distinguished. (i) At low frequencies the values are very scattered, unusually large, and mostly negative. (ii) In the region of diffusons, after a monotonous increase, $\gamma_{a}$ becomes almost constant at $\approx 30$ meV, and its values collapse into a very narrow region. (iii) At locon frequencies $\gamma_{a}$ spreads out again, but much less than at low $\omega$.

Why are the low frequency values of $\gamma$ so large? At most one would expect the data to be scattered between 0 and 1 due to a partial presence of $\mathbf{Q}$ and $\lambda$ for propagons. We find that the cause of this anomalous behavior is the resonant modes. As already shown [21], these modes have very large amplitudes at groups of under-coordinated atoms. Similar groups of over-coordinated atoms are responsible for locons. Since under-coordination implies "softness", it is natural that the internal strain $r_{i \alpha}$ is also largest at these sites. This is why the magnitudes of $\gamma$ are large for the resonant modes. The dispersion of values, also seen for locons, is explained by different nature of topological defects where the modes have largest amplitudes. 
The bands seen in $\gamma(\mathbf{Q}, \lambda)$, Fig. 2(A), of crystalline silicon, are suppressed in the "diffuson" portion of the spectrum of amorphous silicon. This has the following interpretation. In a crystal, knowledge of the pattern of a normal mode in a few adjacent unit cells allows one to predict the pattern in distant regions. In a glass, such a prediction is not possible without a very large computer calculation. More surprising, different normal modes in the "diffuson" part of the spectrum are not globally distinguishable. If one knows the pattern of displacement of two modes ( $a 1$ and $a 2)$ of similar frequencies in one spatial region, one could not recognize which pattern was $a 1$ and which $a 2$ in a distant region. There seem not to be properties other than $\omega_{a}$ which can classify these modes.

Finally, Fig. 3(B) shows $S_{a}$ for amorphous silicon. Note the surprising uniformity of bond-stretching character (see also Ref. [20]), even in the region of propagons where memory of polarization character might have been expected. As in the crystalline case, $S_{a}$ closely follows $\gamma_{a}$, except at low frequencies where the values of $\gamma$ are much more influenced by dynamics than by vibrational pattern.

We thank J. L. Feldman and S. Bickham for stimulating discussions. This work was supported by NSF Grant No. DMR 9417755. 


\section{REFERENCES}

[1] T. H. K. Barron, J. G. Collins, and G. K. White, Adv. Phys. 29, 609 (1980).

[2] Yu. M. Galperin, V. L. Gurevich, and D. A. Parshin, Phys. Rev. B 32, 6873 (1985); Yu. M. Galperin, V. G. Karpov, and V. I. Kozub, Adv. Phys. 38, 669 (1989).

[3] G. K. White, Phys. Rev. Lett. 34, 204 (1975); K. G. Lyon, G. L. Salinger, and C. A. Swenson, Phys. Rev. B 19, 4231 (1979); G. J. Morgan, G. K. White, and J. G. Collins, Phil. Mag. B 43, 1039 (1981); D. A. Ackerman, A. C. Anderson, E. J. Cotts, J. N. Dobbs, W. M. MacDonald, and F. J. Walker, Phys. Rev. B 29, 966 (1984); G. K. White, S. J. Collocott, and J. S. Cook, Phys. Rev. B 29, 4778 (1984); G. A. Saunders, R. D. Metcalfe, M. Cutroni, M. Frederico, and A. Piccolo, Phys. Rev. B 53, 5287 (1996).

[4] A. Witvrouw and F. Spaepen, J. Appl. Phys. 74, 7154 (1993).

[5] G. Dolling and R. A. Cowley, Proc. Phys. Soc. 88, 463 (1966).

[6] R. Vacher, J. Pelous, F. Plicque, and A. Zarembowitch, J. Non-Crys. Sol. 45, 397 (1981); C. J. Morath and H. J. Maris, Phys. Rev. B 54, 203 (1996); B. E. White and R. O. Pohl, Z. Phys. B 100, 401 (1996).

[7] J. Fabian and P. B. Allen (unpublished).

[8] T. H. K. Barron and M. L. Klein, in Dynamical Properties of Solids, edited by G. K. Horton and A. A. Maradudin (North-Holland, Amsterdam, 1974) Vol. I, p. 391.

[9] F. H. Stillinger and T. A. Weber, Phys. Rev. B 31, 5262 (1985).

[10] J. Q. Broughton and X. P. Li, Phys. Rev. B 35, 9120 (1987); X. P. Li, G. Chen, P. B. Allen, and J. Q. Broughton, Phys. Rev. B 38, 3331 (1988); J. L. Feldman, J. Q. Broughton, and F. Wooten, Phys. Rev. B 43, 2152 (1991).

[11] J. L. Feldman, M. Kluge, P. B. Allen, and F. Wooten, Phys. Rev. B 48, 12589 (1993).

[12] J. Fabian and P. B. Allen, Phys. Rev. Lett. 77, 3839 (1996). 
[13] F. Wooten, K. Winer, and D. Weaire, Phys. Rev. Lett. 54, 1392 (1985).

[14] S. Kugler, L. Pusztai, L. Rosta, P. Chieux, and R. Bellissent, Phys. Rev. B 48, 7685 (1993).

[15] A. A. Maradudin, E. W. Montroll, G. H. Weiss, and I. P. Ipatova, Theory of Lattice Dynamics in the Harmonic Approximation, Solid State Physics, Supplement 3 (Academic Press, New York, 1971).

[16] R. E. Cowley, Phys. Rev. Lett. 60, 2379 (1988).

[17] H. Ibach, Phys. Stat. Sol. 31, 625 (1969); G. A. Slack and S. F. Bartram, J. Appl. Phys. 46, 89 (1975).

[18] S. Biernacki and M. Scheffler, Phys. Rev. Lett. 63, 290 (1989); C. Z. Wang, C. T. Chan, and K. M. Ho, Phys. Rev. B 42, 11276 (1990); G. K. White, Contemp. Phys. 34, 193 (1993).

[19] G. K. White, J. Phys. D 6, 2070 (1973).

[20] M. Marinov and N. Zotov, Phys. Rev. B 55, 2938 (1997).

[21] R. Biswas, A. M. Bouchard, W. A. Kamikatahara, G. S. Grest, and C. M. Soukolis, Phys. Rev. Lett. 60, 2280 (1988); H. R. Schober and B. Liard, Phys. Rev. B 44, 6746 (1991); H. R. Schober and C. Oligschleger, Phys. rev. B 53, 11469 (1996).

[22] J. Fabian, J. L. Feldman, and P. B. Allen (unpublished). 
FIG. 1. Calculated and measured linear thermal expansion $\alpha(\mathrm{T})$ for crystalline and amorphous silicon. IS: internal strain.

FIG. 2. Calculated Grüneisen parameters $\gamma_{a}(\mathrm{~A})$ and bond stretching parameters $S_{a}(\mathrm{~B})$ for crystalline silicon as a function of frequency. In (A) the polarization labels are: TA - transverse acoustic, LA - longitudinal acoustic, LO - longitudinal optical, TO - transverse optical. The solid line is the vibrational densities of states (DOS) in arbitrary units.

FIG. 3. Calculated Grüneisen parameters $\gamma_{a}(\mathrm{~A})$ and bond-stretching character $S_{a}(\mathrm{~B})$ of amorphous silicon as a function of frequency. The vertical line at $\omega_{c} \approx 71 \mathrm{meV}$ is the mobility edge. The scale in (A) is split by the horizontal line at -1 to emphasize the large negative values of $\gamma$ at low $\omega$. The solid line is DOS in arbitrary units. 


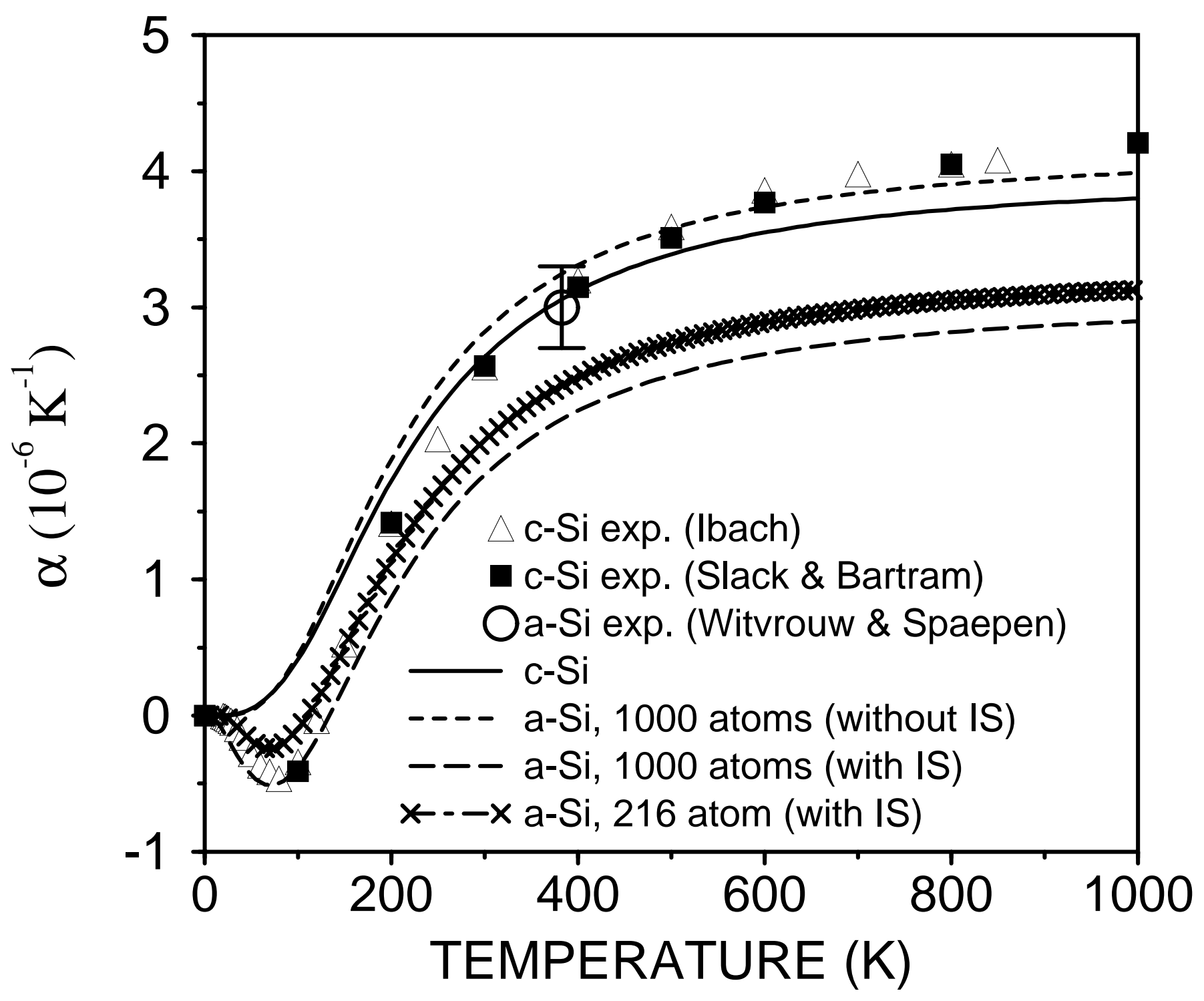




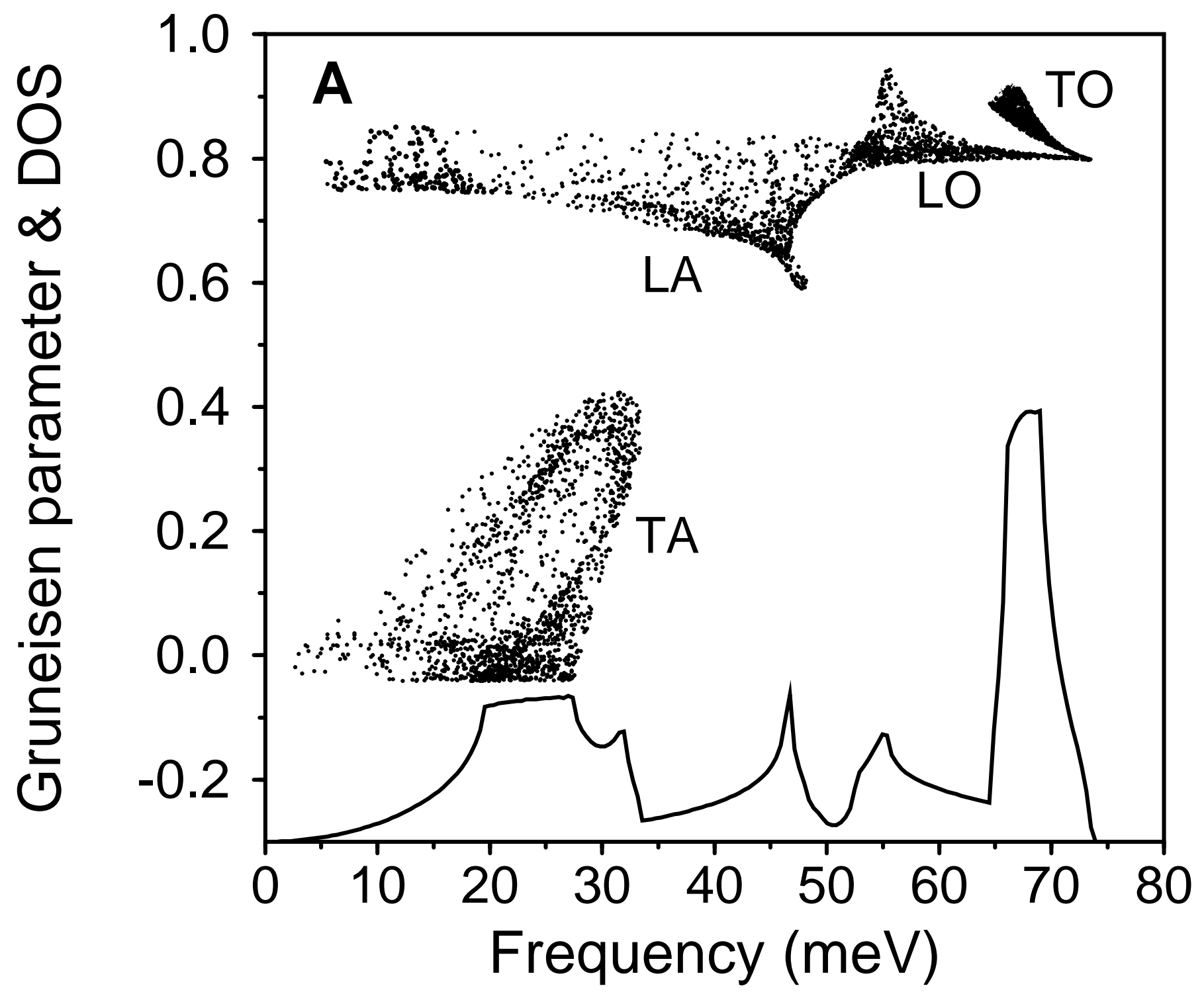




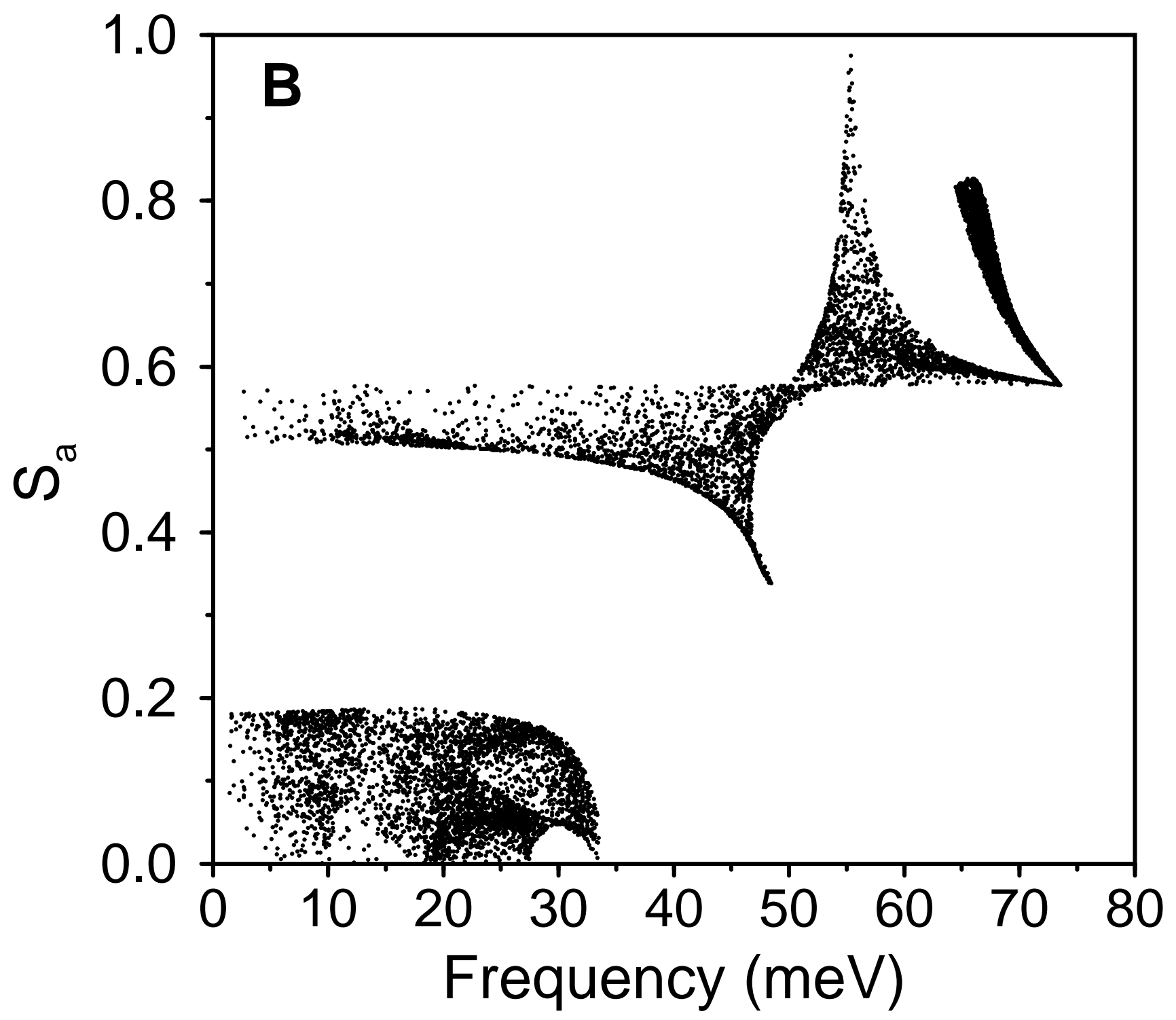



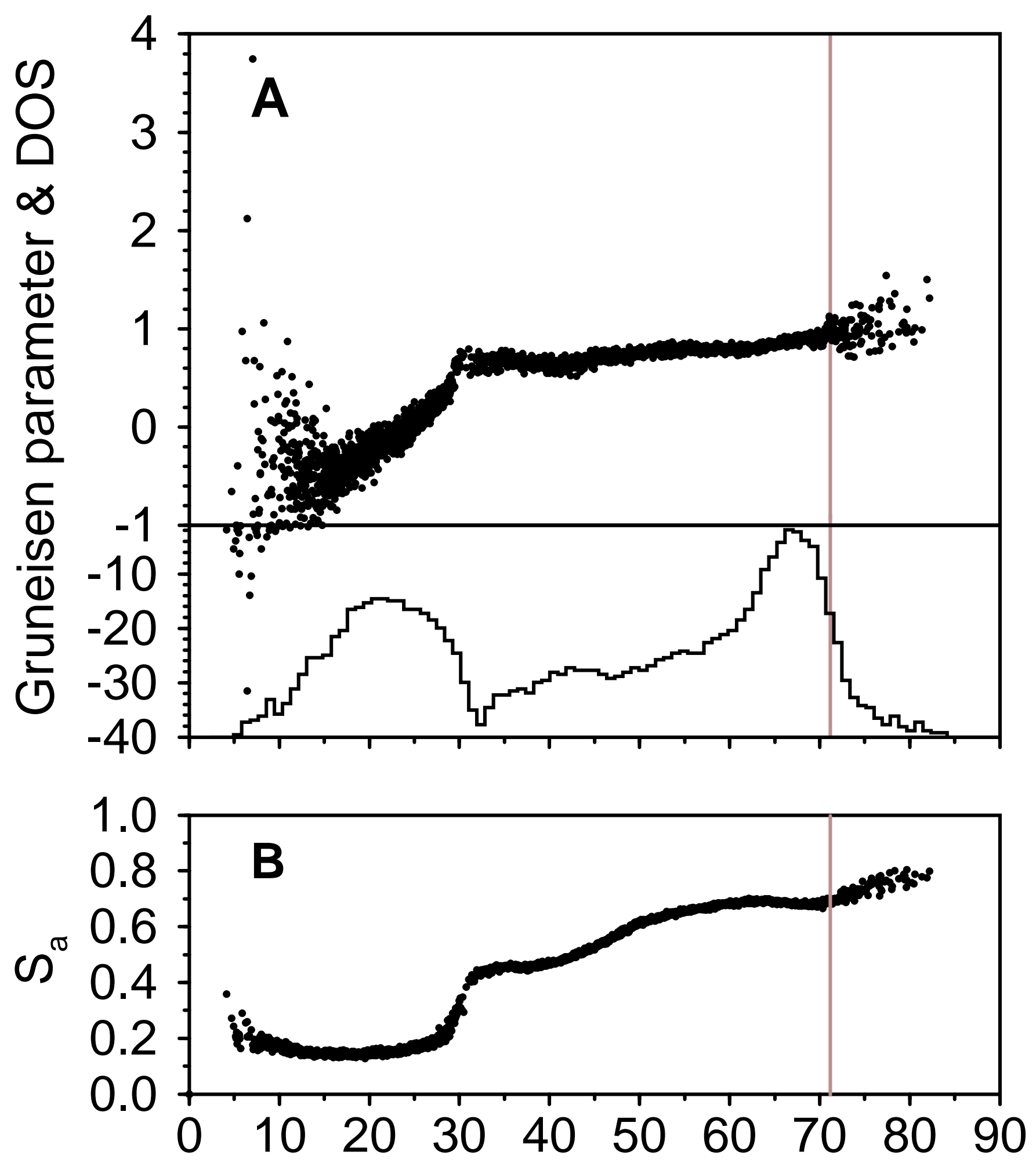